\title{
The solution of linear mechanical systems in terms of path superposition
}

\author{
F.X. Magrans ${ }^{* 1,2}$, J. Poblet-Puig ${ }^{\dagger 2}$ and A. Rodríguez-Ferran ${ }^{\ddagger 2}$ \\ ${ }^{1}$ Ingeniería para el Control del Ruido \\ ${ }^{2}$ Laboratori de Càlcul Numèric, E.T.S. d'Enginyers de Camins, Canals \\ i Ports de Barcelona, Universitat Politècnica de Catalunya
}

August 17, 2016

\begin{abstract}
We prove that the solution of any linear mechanical system can be expressed as a linear combination of signal transmission paths. This is done in the framework of the Global Transfer Direct Transfer (GTDT) formulation for vibroacoustic problems. Transmission paths are expressed as powers of the transfer matrix. The key idea of the proof is to generalise the Neumann series of the transfer matrix -which is convergent only if its spectral radius is smaller than one- into a modified Neumann series that is convergent regardless of the eigenvalues of the transfer matrix. The modification consists in choosing the appropriate combination coefficients for the powers of the transfer matrix in the series. A recursive formula for the computation of these factors is derived. The theoretical results are illustrated by means of numerical examples. Finally, we show that the generalised Neumann series can be understood as an acceleration (i.e. convergence speedup) of the Jacobi iterative method.
\end{abstract}

*correspondence: Berruguete 52, Vila Olímpica (Vall d'Hebron), E-08035 Barcelona, Spain, email: fxmagrans@icrsl.com

${ }^{\dagger}$ correspondence: UPC, Campus Nord B1, Jordi Girona 1, E-08034 Barcelona, Spain, e-mail: jordi.poblet@upc.edu

${ }^{\ddagger}$ correspondence: UPC, Campus Nord C2, Jordi Girona 1, E-08034 Barcelona, Spain, e-mail: antonio.rodriguez-ferran@upc.edu 


\section{List of symbols}

$\begin{array}{ll}\mathbf{A} & \text { system matrix } \\ \alpha_{i}, \beta_{j, i}, \gamma_{i} & \text { parameters in generalised Neumann series } \\ \mathbf{b} & \text { vector of forces or excitations } \\ \mathbf{D} & \text { diagonal part of matrix } \mathbf{A} \\ \mathbf{I} & \text { identity matrix } \\ \mathbf{L} & \text { strict lower triangular part of matrix } \mathbf{A} \\ \lambda & \text { eigenvalues (typically of matrix } \mathbf{T}) \\ n & \text { problem dimension } \\ \mathbf{S}_{j, m} & \text { partial sum of order } j(j \text { modified parameters) and } m+1 \text { terms } \\ \mathbf{T} & \text { transfer matrix } \\ \mathbf{T} & \text { global transfer matrix } \\ \mathbf{U} & \text { strict upper triangular part of matrix } \mathbf{A} \\ \mathbf{x} & \text { vector of unknowns or signals }\end{array}$

\section{Introduction}

Vibroacoustic problems are very often not easy to visualise and understand. Moreover, the availability of experimental measurements is limited by operational costs and times. For these reasons the intuition of engineers/physicists/acousticians has always played an important role during the design process. A key concept is the transmission path of sound and vibrations. This has remained an intuitive idea rather than a properly defined and well established concept.

The first attempts to quantify the contribution of subsystems, even if they never spoke about paths, can be found in [1] and later works [2, 3, 4, 5, 6]. They were motivated by the need in the automotive industry to characterise how the noise generated by the engine or in the moving parts of the vehicle were transmitted to the cabin. This method is nowadays known as Operational Transfer Path Analysis (OTPA), [7, 8].

Paths are implicitly defined and quantified in [9]. The role of paths in more specific situations was analysed in $[10,11]$, where the interest is focused in the characterisation of the connectivity between system parts. This method is known as Global Transfer Direct Transfer (GTDT) method in the papers or as Advanced Transfer Path Analysis (ATPA) in the industry, where it has been widely used in many applications, such as railways [12]. Some academic tests can be also found in [13, 14]. A comprehensive classification of the methods and historical overview can be found in [15]. Paths have also been defined and quantified in a Statistical Energy Analysis (SEA) framework $[16,17,18]$.

Other applications of the path concept can be found in the literature. An analysis of the paths that contribute more to the system response by means of graph theory is presented in $[19,20]$. Path analysis was simplified considering forward paths only in [18]. A comparison of path analysis with other methods was reported in [21].

In spite of the clear applicability of path analysis to practical situations, a theoretical question remains open: a proof of completeness. In other words, the possibility of fully describing the solution of a mechanical transfer problem by means of the superposition of transmission paths. This theoretical question is addressed here, by using 
concepts and tools of numerical linear algebra (see textbooks [22, 23] for background material).

It was clearly demonstrated in $[24,18]$ that a solution of a mechanical problem can be described by means of the Neumann series ${ }^{1}$ of the transfer matrix $\mathbf{T}$ (the powers of $\mathbf{T}$ are a representation of paths of different order in the mechanical system). The series has strict convergence conditions, which in practise mean that the solution description through transmission paths and Neumann series ${ }^{2}$ is not always possible.

The issue of the completeness of the solution description has also been addressed in $[25,26,19,27]$. [25] relates the convergence of the series with the damping of the systems because the energy of undamped systems permanently excited would grow indefinitely. However, this does not explain the situations when, even with damping, the series diverge. For this reason [19] claims that other conditions to ensure the convergence are required in addition to the existence of damping. The drawback of the divergence of the solution expressed as a series also appears if the problem is not strictly formulated in terms of the transfer matrix and its powers. This can be seen in [26] for the matrix of coupling loss factors and in [27] for the coupling eigenvalues.

The goals and achievements of this research are as follows:

- To provide a definition of what a path is.

- To prove the possibility of expressing the solution of all linear problems in terms of paths (especially applied to vibroacoustics). The proof is done in the framework of the Direct and Global Transfer Matrix formulation of the problem. The final result is a generalisation of the Neumann series.

- To derive a practical recursive methodology that allows the computation of the solution based on the transfer matrix of the problem. The goal is also to provide a closed-form expression of the solution as simple as possible.

- To illustrate this methodology with numerical examples.

- To explore the relationship between the proposed approach and Jacobi iterative method for linear systems. The generalised Neumann series can be understood as an acceleration (i.e. convergence speedup) of the Jacobi method.

The remainder of the paper is organised as follows. Some key concepts such as the notion of path and the transfer matrix are defined in Section 2. A precise and explicit definition of path is given. The theoretical core of the research is presented in Section 3. It includes the proof of the existence of an expression of the system solution based on a linear combination of paths. A general methodology to compute the combination factors in the generalised Neumann series is provided. Numerical examples that illustrate the theoretical results are shown in Section 4. The concluding remarks of Section 5 close the paper.

\footnotetext{
${ }^{1}$ In honour of Karl Gottfried Neumann (1832-1925)

${ }^{2} \mathrm{~A}$ Neumann series has the form $\sum_{k=0}^{\infty} \mathbf{T}^{k}$.
} 


\section{Definitions}

\subsection{Physical considerations on the 'path' concept}

Various methods based on path analysis are useful to find engineering solutions in vibroacoustic problems [15]. Nevertheless the 'path' concept is often not defined in a rigorous way.

Our goal here is to provide this explicit definition of 'path'. It will help later in Section 3 in order to develop the core of this research. The definition must be consistent with these three intuitive ideas:

1. A path is something different from a contribution. It must provide information on how the system behaves, instead of being understood as a black box with an input and an output. In techniques such as Transfer Path Analysis (TPA, [28]) the output is a product of multiple contributions that arrive through different uncharacterised paths. This is not the case of ATPA (GTDT) or OTPA, which are based on the path concept.

2. A path is not only defined by the topology of the problem. In addition, the physical behaviour of the system must be considered. Clear examples of this are: i) a beam, where the deflection of a point is linked not only with the deflection of other points but also with the rotations [11]; or ii) an SEA description of the sound transmission between two rooms, where coupling loss factors due to forced or resonant transmissions must be considered in the same single wall [29]. For the case $i$ ) see the simple sketch in Fig. 1 where a supported beam is represented. Three different points in the beam are considered $(i=1,2,3)$. Each of them is characterised by its displacement $x_{i}$. However, this is not enough in order to study the signal transmission between 1 and 3 because an imposed displacement at 1 causes a displacement at 3 even if the displacement at 2 is blocked. The signal can pass from 1 to 3 not only through the displacement in 2 but also through the rotation in 2 . So, two different transmission paths must be considered in order to properly characterise the mechanical system response. It is clearly shown in the path diagram of Fig. 1.

3. A path is the edge of a weighted graph including the physical characteristics of the problem. The quantity that characterises the link between two nodes must be related with the physics of the problem.

\subsection{Definition of paths}

Let $N=\{1,2, \ldots, n\}$ be a set of $n$ nodes. The nodes may represent single points in a continuous system, a degree of freedom associated to that point, single masses in a discrete system of masses and springs or disjoint parts of a continuous system. Their intersection must be null. Let $x_{i}$ be a physical signal and $b_{i}$ an excitation, both associated to node $i$. 


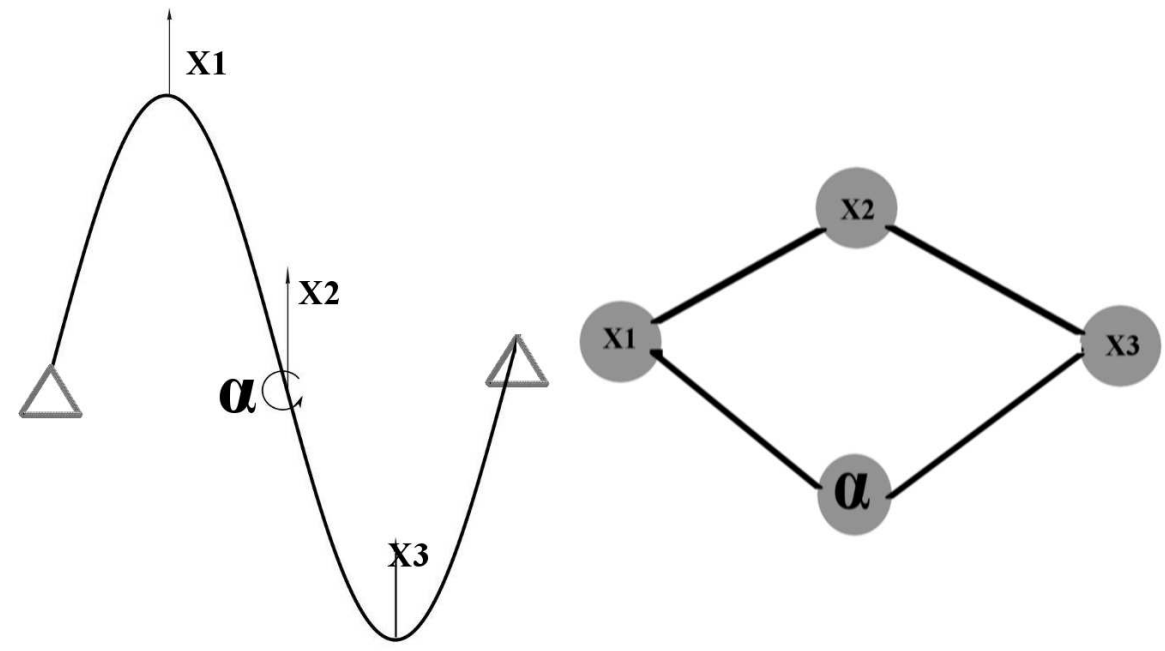

Figure 1: Path representation of a simply supported beam. Four degrees of freedom are considered: three displacements $x_{i}$ and one rotation $\alpha$.

The behaviour of the mechanical system is properly described by the following linear system of equations:

$$
\mathbf{A x}=\mathbf{b} \quad x_{i}, b_{i}, a_{i j} \in \mathbb{C}
$$

A direct path between nodes $i$ and $j$ exists and is represented by coefficient $p_{i j}$ if the following conditions are satisfied:

1. $p_{i j} \neq 0$ links signals $x_{i}$ and $x_{j}$ caused by an excitation in node $i$ while all other nodes are blocked:

$$
x_{j}=p_{i j} x_{i} \quad \forall j \neq i, \text { for } x_{k}=0, \forall k \neq i, j, b_{i} \neq 0 \text { and } b_{j}=0
$$

The non-zero coefficient $p_{i j}$ will be called direct transfer between nodes $i$ and $j$.

2. It is possible to express the solution of the system as a superposition of paths of arbitrary order.

A $k$-order path $p_{i j}^{(k)}$ is as a chain of $k$ direct paths that starts at node $i$ and ends at node $j$ with $j \neq i$ or $j=i$ such that: $p_{i j}^{(k)}=\underbrace{p_{i r} p_{r s} \ldots p_{q j}}_{k}$.

That is, it must be possible to express every signal $x_{j}$ as

$$
x_{j}=\sum_{i=1}^{n} \sum_{\substack{\text { all paths } \\ k=1}}^{\infty} \gamma_{k} p_{i j}^{(k)} x_{i}
$$

where $\gamma_{k}$ are combination factors that depend on the order of the path (see Section 3 where $\gamma_{k}$ are defined in Eq. (42)). 
Several models used to describe the response of mechanical systems can fit in the definition of the path concept presented here, see for example [9, 26, 27, 30].

\subsection{The Global Transfer Direct Transfer (GTDT) frame for paths}

The basic concepts of the GTDT method [9] are briefly reviewed here.

\subsubsection{Problem statement}

Consider the linear system of equations (1), where $\mathbf{A}$ is a (in general complex but possibly real) invertible matrix of dimension $n$. Matrix $\mathbf{A}$ may be expressed as

$$
\mathbf{A}=\mathbf{D}+\mathbf{L}+\mathbf{U}
$$

where $\mathbf{D}, \mathbf{L}$ and $\mathbf{U}$ are the diagonal, strict lower and strict upper parts of matrix $\mathbf{A}$. By assuming that $\mathbf{D}$ is invertible, system (1) may be recast as

$$
\mathbf{x}=\mathbf{D}^{-1} \mathbf{b}+\mathbf{T x}
$$

where

$$
\mathbf{T}=-\mathbf{D}^{-1}(\mathbf{L}+\mathbf{U})
$$

is the transfer matrix. This matrix is the transpose of the Direct Transfer Matrix defined in [9] with zeros in the diagonal. Note that $\mathbf{T}$ is not symmetrical in general and that it takes into account the coupling between the unknowns: for $\mathbf{T}=\mathbf{0}$, the system is diagonal and the solution is simply $\mathbf{x}=\mathbf{D}^{-1} \mathbf{b}$.

From Eq. (5), the solution of system (1) may be expressed as

$$
\mathbf{x}=(\mathbf{I}-\mathbf{T})^{-1} \mathbf{D}^{-1} \mathbf{b}
$$

Finally, we define the Global Transfer Matrix

$$
\mathbf{T}^{G}=(\mathbf{I}-\mathbf{T})^{-1} \mathbf{D}^{-1}
$$

Note that the definition of the Global Transfer Matrix in [9] contains a diagonal scaling matrix not included here. This allows a change between forces and displacements as main variables. In an experimental research it is often more advantageous to work with displacements because they can be measured more easily. On the contrary, in the context of the present work it is more convenient to think in terms of a forcedisplacement formulation. This diagonal scaling matrix implies that the coefficients of $\mathbf{T}^{G}$ have the physical meaning of receptance Frequency Response Functions (FRF).

\subsubsection{Transfer matrices: physical meaning}

Note that, under the assumptions of Eq. (2), equation $j$ of the linear system (5) is

$$
x_{j}=t_{j i} x_{i} \quad \forall j \neq i, \text { for } x_{k}=0, \forall k \neq i, j, b_{i} \neq 0 \text { and } b_{j}=0
$$


where $t_{j i}$ are the entries of matrix $\mathbf{T}$. This means that matrix $\mathbf{T}$ contains all the information on the direct signal transmission from one node to another when all the rest are blocked. This the first requirement in Section 2.2. The main goal of this research is to prove that matrix $\mathbf{T}$ also satisfies the second requirement.

The formulation of the problem in terms of the transfer matrix is valid for the modelling of mechanical systems by means of different techniques such as: i) Statistical Energy Analysis [29], in which case the system matrix A is created from the coupling loss factors that relate the subsystems and their damping coefficient; ii) the Finite Element Method (FEM [31]), in which case the system matrix $\mathbf{A}=\mathbf{K}-\omega^{2} \mathbf{M}$, where $\mathbf{K}$ and $\mathbf{M}$ are the stiffness and mass matrices (damping can also be considered); or iii) models or methods where $\mathbf{T}$ can be indirectly obtained by means of experimental measurements in a laboratory as described in [9].

Matrix $\mathbf{T}$ contains information of the system connectivity and its successive powers are a representation of the $k$-order paths. This can be deduced from the graph theory and the properties of the powers of the adjacency matrix, see for example [32].

The recursive substitution of Eq. (5) in itself leads to the following identity:

$$
\mathbf{x}=\left(\mathbf{I}+\mathbf{T}+\mathbf{T}^{2}+. .+\mathbf{T}^{k-1}\right) \mathbf{D}^{-1} \mathbf{b}+\mathbf{T}^{k} \mathbf{x}
$$

This makes evident the relationship between the Neumann series, the re-formulation of the problem in terms of the transfer matrix and the solution of the original linear problem. When the spectral radius (i.e. largest modulus of eigenvalues, see [33, 22, 23]) of $\mathbf{T}$ is less than one, the limit for $k$ tending to infinity of vector $\mathbf{T}^{k} \mathbf{X}$ is zero and the solution of the problem is the Neumann series. In other words, the solution of the problem is the simplest (all combination coefficients equal one) linear combination of $k$-order paths. The order $k$ starts at zero and the series makes sense when $k$ tends to infinity. This case is more rigorously considered later in Section 3.1.

The coefficients of the Global Transfer Matrix $\mathbf{T}^{G}$, as defined in Eq. (8), express the signal in node $j$ exciting the node $i$ when the rest of the nodes are not blocked. Consequently, the signal at node $j$ includes the contributions of all the paths. $\mathbf{T}^{G}$ is more easily measurable in the laboratory than $\mathbf{T}$, which must be indirectly measured by means of laborious tests like the strip method [34].

Finally, matrix $\mathbf{D}^{-1}$ is the matrix of direct transfers from node $i$ to node $i$ : $d_{i i}$. It accounts for the part of the signal in node $i$ that does not come from the other nodes but from the external applied forces.

Eq. (5) can be premultiplied by $\mathbf{x}^{t} \mathbf{D}$ leading to

$$
\mathbf{x}^{t} \mathbf{D} \mathbf{x}=\mathbf{x}^{t} \mathbf{b}+\mathbf{x}^{t} \mathbf{D} \mathbf{T} \mathbf{x}
$$

This new expression can be interpreted in energetic terms. The left-hand-side represents the kinetic and potential energy of individual elements and $\mathbf{x}^{t} \mathbf{b}$ is the external work. Consequently, the transfer matrix $\mathbf{T}$ plays the role of an interaction potential. 


\section{Solution based on paths superposition: mathe- matical proof}

The mathematical proof for the existence of a linear combination of paths that leads to the solution of the problem is given here. The methodology is based on the framework presented in Section 2, so, the proof is valid for a wide variety of models that satisfy a very usual algebraic structure: linear system of equations reformulated in terms of the transfer matrix.

In those cases the mathematical expression of path addition is Eq. (10). However, the convergence of the series cannot always be ensured. It depends on very different factors related with the physical properties or the modelling technique, such as the damping distribution in the mechanical system, how are the SEA subsystems defined, which points are chosen in order to define a transfer matrix, etc. Divergence is found if the spectral radius of the transfer matrix is not less than one, $\rho(\mathbf{T}) \nless 1$. This situation, which in fact is the motivation of the discussion and the main goal of the research, is analysed in Section 3.2.

The main question to be answered here, linked with the second aspect of the path definition in Section 2.2, is if it is possible to express $(\mathbf{I}-\mathbf{T})^{-1}$ (and, hence, the solution $\mathbf{x}$ ) as a linear combination of powers of the transfer matrix $\mathbf{T}$ also in the case when the spectral radius of $\mathbf{T}$ is larger than one. This non-trivial case will be analysed afterwards in Section 3.2.

\subsection{Some useful well-known results}

\section{Theorem}

Let $\mathbf{B}$ be a square matrix. The following conditions are equivalent (see for example Theorem 1.5-1 in [33]):

1. $\lim _{k \rightarrow \infty} \mathbf{B}^{k}=\mathbf{0}$

2. $\lim _{k \rightarrow \infty} \mathbf{B}^{k} \mathbf{v}=\mathbf{0}$ for any vector $\mathbf{v}$

3. $\rho(\mathbf{B})<1$, where $\rho(\cdot)$ is the spectral radius

Neumann series, spectral radius less than one

If $\mathbf{T}$ verifies the conditions above, then

$$
(\mathbf{I}-\mathbf{T})^{-1}=\sum_{k=0}^{\infty} \mathbf{T}^{k}
$$

To show this, we consider the partial sum $\mathbf{S}_{0, m}=\sum_{k=0}^{m} \mathbf{T}^{k}$. Subscript $m$ denotes the upper limit of the summation, whereas subscript 0 indicates that it is the original 
Neumann series. Then,

$$
\lim _{m \rightarrow \infty}(\mathbf{I}-\mathbf{T}) \mathbf{S}_{0, m}=\lim _{m \rightarrow \infty}\left(\sum_{k=0}^{m} \mathbf{T}^{k}-\sum_{k=0}^{m} \mathbf{T}^{k+1}\right)=\mathbf{I}-\lim _{m \rightarrow \infty} \mathbf{T}^{m+1}=\mathbf{I}
$$

Note that for $\rho(\mathbf{T})<1$,

$$
\lim _{m \rightarrow \infty} \mathbf{S}_{0, m}=(\mathbf{I}-\mathbf{T})^{-1}
$$

\subsection{Transfer matrices with spectral radius larger than one}

The remainder of the section shows that Eq. (12) can be always generalised to deal with transfer matrices $\mathbf{T}$ such that $\rho(\mathbf{T}) \nless 1$. In other words, the solution of a linear system of equations representing a mechanical system can be expressed as a linear combination of paths of different order as defined in Section 2.3.

\subsubsection{One-parameter modification}

Consider the sequence of matrices $\left\{\mathbf{P}_{1, k}\right\}$ with

$$
\mathbf{P}_{1,0}=\alpha_{1} \mathbf{T}^{0} \text { and } \mathbf{P}_{1, k}=\left(1-\alpha_{1}\right) \mathbf{T}^{k-1}+\alpha_{1} \mathbf{T}^{k} \text { for } k \geq 1
$$

where $\alpha_{1}$ is a free parameter to be determined later, and the modified partial sum

$$
\begin{aligned}
\mathbf{S}_{1, m} & =\sum_{k=0}^{m} \mathbf{P}_{1, k}=\alpha_{1} \mathbf{T}^{0}+\left(1-\alpha_{1}\right) \mathbf{T}^{0}+\alpha_{1} \mathbf{T}^{1}+\cdots+\left(1-\alpha_{1}\right) \mathbf{T}^{m-1}+\alpha_{1} \mathbf{T}^{m} \\
& =\mathbf{S}_{0, m-1}+\alpha_{1} \mathbf{T}^{m}
\end{aligned}
$$

Note that, for $\alpha_{1}=1$, one retrieves the Neumann series (12). The question here is to know, for the case $\alpha_{1} \neq 1$, under what conditions does the partial sum (16) converge to the inverse of $(\mathbf{I}-\mathbf{T})$ :

$$
\begin{aligned}
\lim _{m \rightarrow \infty}(\mathbf{I}-\mathbf{T}) \mathbf{S}_{1, m} & =\lim _{m \rightarrow \infty}\left(\mathbf{S}_{1, m}-\mathbf{T} \mathbf{S}_{1, m}\right) \\
& =\lim _{m \rightarrow \infty}\left(\mathbf{S}_{0, m-1}+\alpha_{1} \mathbf{T}^{m}-\mathbf{T} \mathbf{S}_{0, m-1}-\alpha_{1} \mathbf{T}^{m+1}\right) \\
& =\mathbf{I}-\lim _{m \rightarrow \infty}\left(\left(1-\alpha_{1}\right) \mathbf{T}^{m}+\alpha_{1} \mathbf{T}^{m+1}\right)
\end{aligned}
$$

The equality $(\mathbf{I}-\mathbf{T}) \mathbf{S}_{0, m-1}=\mathbf{I}-\mathbf{T}^{m}$ is used for the last step. Eq. (17) shows that $\mathbf{S}_{1, m}$ converges to $(\mathbf{I}-\mathbf{T})^{-1}$ if and only if

$$
\lim _{m \rightarrow \infty}\left(\left(1-\alpha_{1}\right) \mathbf{T}^{m}+\alpha_{1} \mathbf{T}^{m+1}\right)=\lim _{m \rightarrow \infty} \mathbf{P}_{1, m+1}=\mathbf{0}
$$

This condition is less restrictive (and includes) the usual condition $\lim _{m \rightarrow \infty} \mathbf{T}^{m+1}=\mathbf{0}$. 


\subsubsection{Two-parameter modification}

The modification strategy of section 3.2.1 can be applied again. Consider the sequence of matrices $\left\{\mathbf{P}_{2, k}\right\}$ with

$$
\mathbf{P}_{2,0}=\alpha_{2} \mathbf{P}_{1,0} \text { and } \mathbf{P}_{2, k}=\left(1-\alpha_{2}\right) \mathbf{P}_{1, k-1}+\alpha_{2} \mathbf{P}_{1, k} \text { for } k \geq 1
$$

and the modified partial sum

$$
\mathbf{S}_{2, m}=\sum_{k=0}^{m} \mathbf{P}_{2, k}=\mathbf{S}_{1, m-1}+\alpha_{2} \mathbf{P}_{1, m}
$$

The question here is to know under what conditions does the partial sum (20) converge to the inverse of $(\mathbf{I}-\mathbf{T})$ :

$$
\lim _{m \rightarrow \infty}(\mathbf{I}-\mathbf{T}) \mathbf{S}_{2, m}=\lim _{m \rightarrow \infty}\left(\mathbf{S}_{2, m}-\mathbf{T} \mathbf{S}_{2, m}\right)=\mathbf{I}-\lim _{m \rightarrow \infty} \mathbf{P}_{2, m+1}
$$

To obtain the second equality in Eq. (21), one takes into account the definition of the partial sums $\mathbf{S}_{0, m}, \mathbf{S}_{1, m}$ and $\mathbf{S}_{2, m}$. Eq. (21) shows that $\mathbf{S}_{2, m}$ converges to $(\mathbf{I}-\mathbf{T})^{-1}$ if and only if

$$
\lim _{m \rightarrow \infty} \mathbf{P}_{2, m+1}=\lim _{m \rightarrow \infty}\left(\left(1-\alpha_{2}\right) \mathbf{P}_{1, m}+\alpha_{2} \mathbf{P}_{1, m+1}\right)=\mathbf{0}
$$

This condition is less restrictive (and includes, for $\alpha_{2}=1$ ) the condition $\lim _{m \rightarrow \infty} \mathbf{P}_{1, m+1}=$ $\mathbf{0}$ derived in section 3.2.1.

\subsubsection{Multi-parameter modification}

In fact, the modification strategy can be applied recursively. Consider the sequence of matrices $\left\{\mathbf{P}_{j, k}\right\}$ with

$$
\mathbf{P}_{j, 0}=\alpha_{j} \mathbf{P}_{j-1,0} \text { and } \mathbf{P}_{j, k}=\left(1-\alpha_{j}\right) \mathbf{P}_{j, k-1}+\alpha_{j} \mathbf{P}_{j-1, k} \text { for } k \geq 1
$$

and the modified partial sum

$$
\mathbf{S}_{j, m}=\sum_{k=0}^{m} \mathbf{P}_{j, k}=\mathbf{S}_{j-1, m-1}+\alpha_{j} \mathbf{P}_{j-1, m}
$$

The following limit is considered to show under what conditions does the partial sum (24) converge to the inverse of $(\mathbf{I}-\mathbf{T})$ :

$$
\lim _{m \rightarrow \infty}(\mathbf{I}-\mathbf{T}) \mathbf{S}_{j, m}=\lim _{m \rightarrow \infty}\left(\mathbf{S}_{j, m}-\mathbf{T} \mathbf{S}_{j, m}\right)=\mathbf{I}-\lim _{m \rightarrow \infty} \mathbf{P}_{j, m+1}
$$

Eq. (25) shows that $\mathbf{S}_{j, m}$ converges to $(\mathbf{I}-\mathbf{T})^{-1}$ if and only if

$$
\lim _{m \rightarrow \infty} \mathbf{P}_{j, m+1}=\lim _{m \rightarrow \infty}\left(\left(1-\alpha_{j}\right) \mathbf{P}_{j-1, m}+\alpha_{j} \mathbf{P}_{j-1 m+1}\right)=\mathbf{0}
$$

For the reasons discussed next, this recursion is applied at most $n$ times, where $n$ is the problem dimension. 


\subsubsection{Selection of parameters}

Once the less restrictive convergence conditions of Eqs. (18), (22) and (26) are available, an strategy to select the optimal values of the parameters $\alpha_{1}, \alpha_{2}, \ldots, \alpha_{n}$ is required.

Consider the basis $\left\{\mathbf{u}_{i}\right\}_{i=1}^{n}$ of eigenvectors of matrix $\mathbf{T}$, associated to eigenvalues $\left\{\lambda_{i}\right\}_{i=1}^{n}$ (over the complex field $\mathbb{C}$, any matrix $\mathbf{T}$ either $i$ ) diagonalises or $i$ ) is arbitrarily close to one with distinct eigenvalues that does).

By expressing an arbitrary vector $\mathbf{v}$ (see condition 2 in theorem of section 3.1) in this eigenvector basis, $\mathbf{v}=a_{1} \mathbf{u}_{1}+a_{2} \mathbf{u}_{2}+\cdots a_{n} \mathbf{u}_{n}$, one gets

$$
\mathbf{T}^{k} \mathbf{v}=a_{1} \lambda_{1}^{k} \mathbf{u}_{1}+a_{2} \lambda_{2}^{k} \mathbf{u}_{2}+\cdots a_{n} \lambda_{n}^{k} \mathbf{u}_{n}
$$

If $\left|\lambda_{i}\right|<1$ for $i=1, \ldots, n$, then theorem in section 3.1 applies. Assume now that eigenvalue $\lambda_{1}$ violates this condition, $\left|\lambda_{1}\right|>1$, so the Neumann series does not converge. Then, for the one-parameter modification of section 3.2.1,

$$
\mathbf{P}_{1, m+1} \mathbf{v}=a_{1}\left(\left(1-\alpha_{1}\right)+\alpha_{1} \lambda_{1}\right) \lambda_{1}^{m} \mathbf{u}_{1}+\cdots a_{n}\left(\left(1-\alpha_{1}\right)+\alpha_{1} \lambda_{n}\right) \lambda_{n}^{m} \mathbf{u}_{n}
$$

To cancel out the divergent term $\lambda_{1}^{m} \mathbf{u}_{1}$, the accompanying scalar should be zero:

$$
\left(1-\alpha_{1}\right)+\alpha_{1} \lambda_{1}=0 \Longrightarrow \alpha_{1}=\frac{1}{1-\lambda_{1}}
$$

By applying the same argument recursively for each additional eigenvalue that violates the constraint, one gets

$$
\alpha_{1}=\frac{1}{1-\lambda_{1}} ; \alpha_{2}=\frac{1}{1-\lambda_{2}} ; \cdots ; \alpha_{n}=\frac{1}{1-\lambda_{n}}
$$

Remarks:

1. If $\left|\lambda_{i}\right|<1$, the modification step with coefficient $\alpha_{i}$ is not strictly necessary for convergence, but it does accelerate the convergence. This can also be seen in Appendix A.

2. In fact, $\alpha_{i}=1 /\left(1-\lambda_{i}\right)$ are the eigenvalues of $(\mathbf{I}-\mathbf{T})^{-1}$; it is not surprising that incorporating this information into the iterative algorithm improves the convergence.

\subsection{Analysis of different cases}

\subsubsection{Real transfer matrix}

If the transfer matrix $\mathbf{T}$ is real, then its eigenvalues are either real or complex conjugate. Note that, in any case, matrices $\mathbf{I}-\mathbf{T}$ and $(\mathbf{I}-\mathbf{T})^{-1}$ are real.

All eigenvalues real If $\left\{\lambda_{i}\right\}_{i=1}^{n} \in \mathbb{R}$, then $\left\{\alpha_{i}\right\}_{i=1}^{n} \in \mathbb{R}$, and the weighted sum of powers of $\mathbf{T}$ indeed results in a real matrix $(\mathbf{I}-\mathbf{T})^{-1}$. 
Some complex conjugate eigenvalues Assume now that matrix $\mathbf{T}$ has one pair of complex conjugate eigenvalues, $\lambda_{2}=\bar{\lambda}_{1}$. Then the corresponding factors are also complex conjugate, $\alpha_{2}=\bar{\alpha}_{1}$. The two-parameter modification of section 3.2.2 results in

$$
\begin{aligned}
\mathbf{S}_{2, m} & =\mathbf{S}_{1, m-1}+\alpha_{2} \mathbf{P}_{1, m}=\mathbf{S}_{0, m-2}+\alpha_{1} \mathbf{T}^{m-1}+\alpha_{2}\left(\left(1-\alpha_{1}\right) \mathbf{T}^{m-1}+\alpha_{1} \mathbf{T}^{m}\right) \\
& =\sum_{k=0}^{m-2} \mathbf{T}^{k}+\left[\alpha_{1}+\alpha_{2}\left(1-\alpha_{1}\right)\right] \mathbf{T}^{m-1}+\alpha_{2} \alpha_{1} \mathbf{T}^{m} \\
& =\sum_{k=0}^{m-2} \mathbf{T}^{k}+2 \frac{1-\Re\left(\lambda_{1}\right)}{1-2 \Re\left(\lambda_{1}\right)+\left|\lambda_{1}\right|^{2}} \mathbf{T}^{m-1}+\frac{1}{1-2 \Re\left(\lambda_{1}\right)+\left|\lambda_{1}\right|^{2}} \mathbf{T}^{m}
\end{aligned}
$$

Note that, as expected, the weights are again real. The same argument applies if $\mathbf{T}$ has more pairs of complex conjugate eigenvalues.

\subsubsection{Complex transfer matrix}

If the transfer matrix $\mathbf{T}$ is complex, all the relevant objects are also complex (i.e. the eigenvalues $\lambda_{i}$, the parameters $\alpha_{i}$, the weights in the weighted sum of powers of $\mathbf{T}$, matrix $\mathbf{I}-\mathbf{T}$ and its inverse...).

\subsection{Recursive method}

The modified partial sums of order $j$ can be computed once the modified partial sums of order $j-1$ and the correction parameter $\alpha_{j}$ are known. From the definition in Eq. (16),

$$
\mathbf{S}_{j-1, m}=\sum_{k=0}^{m-1} \mathbf{P}_{j-1, k}+\mathbf{P}_{j-1, m}=\mathbf{S}_{j-1, m-1}+\mathbf{P}_{j-1, m}
$$

from where $\mathbf{P}_{j-1, m}$ can be obtained and substituted in the definition of $\mathbf{S}_{j, m}$ in Eq. (24):

$$
\mathbf{S}_{j, m}=\alpha_{j} \mathbf{S}_{j-1, m}+\left(1-\alpha_{j}\right) \mathbf{S}_{j-1, m-1}
$$

\subsection{Explicit expression}

An explicit expression of the solution can be obtained now by means of the repeated use of Eq. (33). It must be first particularised for $j=1$,

$$
\mathbf{S}_{1, m}=\alpha_{1} \mathbf{S}_{0, m}+\left(1-\alpha_{1}\right) \mathbf{S}_{0, m-1}
$$

For $j=2$ one gets

$$
\begin{aligned}
& \mathbf{S}_{2, m}=\alpha_{2} \mathbf{S}_{1, m}+\left(1-\alpha_{2}\right) \mathbf{S}_{1, m-1} \\
& =\alpha_{1} \alpha_{2} \mathbf{S}_{0, m}+\left[\alpha_{1}\left(1-\alpha_{2}\right)+\left(1-\alpha_{1}\right) \alpha_{2}\right] \mathbf{S}_{0, m-1}+\left(1-\alpha_{1}\right)\left(1-\alpha_{2}\right) \mathbf{S}_{0, m-2}
\end{aligned}
$$

and, for $j=3$

$$
\mathbf{S}_{3, m}=\beta_{3,0} \mathbf{S}_{0, m}+\beta_{3,1} \mathbf{S}_{0, m-1}+\beta_{3,2} \mathbf{S}_{0, m-2}+\beta_{3,3} \mathbf{S}_{0, m-3}
$$


where

$$
\begin{aligned}
& \beta_{3,0}=\alpha_{1} \alpha_{2} \alpha_{3} \\
& \beta_{3,1}=\alpha_{1} \alpha_{2}\left(1-\alpha_{3}\right)+\alpha_{1}\left(1-\alpha_{2}\right) \alpha_{3}+\left(1-\alpha_{1}\right) \alpha_{2} \alpha_{3} \\
& \beta_{3,2}=\alpha_{1}\left(1-\alpha_{2}\right)\left(1-\alpha_{3}\right)+\left(1-\alpha_{1}\right) \alpha_{2}\left(1-\alpha_{3}\right)+\left(1-\alpha_{1}\right)\left(1-\alpha_{2}\right) \alpha_{3} \\
& \beta_{3,3}=\left(1-\alpha_{1}\right)\left(1-\alpha_{2}\right)\left(1-\alpha_{3}\right)
\end{aligned}
$$

Inspecting the structure of Eqs. (35), (36) and (37) carefully, it is possible to derive a generic expression for $\mathbf{S}_{j, m}$ and coefficients $\beta_{j, i}$ :

$$
\mathbf{S}_{j, m}=\sum_{i=0}^{j} \beta_{j, i} \mathbf{S}_{0, m-i}
$$

with $\beta_{j, i}$ expressed, in multi-index notation, as

$$
\beta_{j, i}=\sum_{k=1}^{\left(\begin{array}{l}
j \\
i
\end{array}\right)} \boldsymbol{\alpha}^{\boldsymbol{\omega}_{k}}(\mathbf{1}-\boldsymbol{\alpha})^{\mathbf{1}-\boldsymbol{\omega}_{k}}
$$

Eq. (39) has a conveniently compact expression thanks to the use of multi-index notation. For instance, for coefficient $\beta_{3,1}$ shown in Eq. (37),

$$
\begin{aligned}
\beta_{3,1} & =\alpha_{1}^{1} \alpha_{2}^{1} \alpha_{3}^{0}\left(1-\alpha_{1}\right)^{0}\left(1-\alpha_{2}\right)^{0}\left(1-\alpha_{3}\right)^{1} \\
& +\alpha_{1}^{1} \alpha_{2}^{0} \alpha_{3}^{1}\left(1-\alpha_{1}\right)^{0}\left(1-\alpha_{2}\right)^{1}\left(1-\alpha_{3}\right)^{0} \\
& +\alpha_{1}^{0} \alpha_{2}^{1} \alpha_{3}^{1}\left(1-\alpha_{1}\right)^{1}\left(1-\alpha_{2}\right)^{0}\left(1-\alpha_{3}\right)^{0} \\
& =\boldsymbol{\alpha}^{\boldsymbol{\omega}_{1}}(\mathbf{1}-\boldsymbol{\alpha})^{\mathbf{1}-\boldsymbol{\omega}_{1}}+\boldsymbol{\alpha}^{\boldsymbol{\omega}_{2}}(\mathbf{1}-\boldsymbol{\alpha})^{\mathbf{1 - \boldsymbol { \omega } _ { 2 }}}+\boldsymbol{\alpha}^{\boldsymbol{\omega}_{3}}(\mathbf{1}-\boldsymbol{\alpha})^{\mathbf{1 - \boldsymbol { \omega } _ { 3 }}}
\end{aligned}
$$

with $\boldsymbol{\alpha}=\left(\alpha_{1}, \alpha_{2}, \alpha_{3}\right)$ and $\boldsymbol{\omega}_{1}=(1,1,0), \boldsymbol{\omega}_{2}=(1,0,1), \boldsymbol{\omega}_{3}=(0,1,1)$.

Finally, Eq. (38) can be reorganised in order to express the partial sum in terms of the powers of the transfer matrix $\mathbf{T}$. This is, in fact, the main goal of the research: to show that the solution of the mechanical problem can be expressed as a linear combination of transmission paths. Indeed,

$$
\mathbf{S}_{j, m}=\sum_{k=0}^{m-j} \mathbf{T}^{k}+\sum_{k=m-j+1}^{m} \gamma_{m-k} \mathbf{T}^{k}
$$

with

$$
\gamma_{p}=\sum_{i=0}^{p} \beta_{j, i} \quad p=0, \ldots, j-1
$$

Note that the first $m-j+1$ terms of $S_{j, m}$ are those of the original Neumann series, whereas the last $j$ terms are weighted with the correction factors $\gamma$. These modifications are the higher-order paths.

A possible physical interpretation of Eq. (41) is that the first sum (lower-order paths) represents the action of the forces on the system, while the second sum (corrected higher-order paths) represents the reaction of the system. The important aspect here is that both response types have been expressed as addition of paths within the 
system. The whole Eq. (41) represents the steady equilibrium state of the mechanical system. At that point the external excitation and the movement of the mechanical system are coordinated in such a way that the excitation does not cause an increase of response.

If the spectral radius is larger than one, it leads to two infinites that compensate each other. If the spectral radius is less than one, the second sum is not relevant anymore. This agrees well with the fact that if the response is controlled by damping (which in general leads to spectral radius less than one), the high-order paths can be neglected because energy is lost in the passage through every path. In terms of Eq. (41) this means that the correction (second sum) is not required to ensure convergence.

If $j=n$ terms of the series are modified ( $n$ is the problem dimension), the series convergence is ensured,

$$
\lim _{m \rightarrow \infty} \mathbf{S}_{n, m}=(\mathbf{I}-\mathbf{T})^{-1}
$$

This is because in the worst scenario all the matrix eigenvalues have a module larger than one. In this critical situation $n$ modified terms of the series need to be added in order to treat the $n$ eigenvalues. In practice, the inverse of $(I-T)$ is approximated by the partial sum $S_{n, m}$ for a finite value of $m$.

\section{$4 \quad$ Numerical examples}

The results of Section 3 are illustrated here by means of the simple system of Fig. 2 . It is a one-dimensional mechanism composed of eight masses: $m_{1}=100 \mathrm{~kg}, m_{2}=200$ $\mathrm{kg}, m_{3}=300 \mathrm{~kg}, m_{4}=400 \mathrm{~kg}, m_{5}=500 \mathrm{~kg}, m_{6}=630 \mathrm{~kg}, m_{7}=700 \mathrm{~kg}, m_{8}=800$ kg. They are linked by means of springs with stiffnesses: $k_{1}=10^{9} \mathrm{~N} / \mathrm{m}, k_{2}=310^{9}$ $\mathrm{N} / \mathrm{m}, k_{3}=1.110^{9} \mathrm{~N} / \mathrm{m}, k_{4}=410^{9} \mathrm{~N} / \mathrm{m}, k_{5}=10^{9} \mathrm{~N} / \mathrm{m}, k_{6}=510^{9} \mathrm{~N} / \mathrm{m}, k_{7}=10^{9}$ $\mathrm{N} / \mathrm{m}, k_{8}=310^{9} \mathrm{~N} / \mathrm{m}$ and $k_{9}=410^{9} \mathrm{~N} / \mathrm{m}$. The frequencies at which matrix $\mathbf{D}$ is singular are listed in Table 1. They can be understood as the vibration frequencies of each single mass when all the others are blocked. Table 1 also shows the coupled eigenfrequencies of the mechanical system (matrix $\mathbf{A}$ ). The dynamic stiffness matrix of the mechanical system is

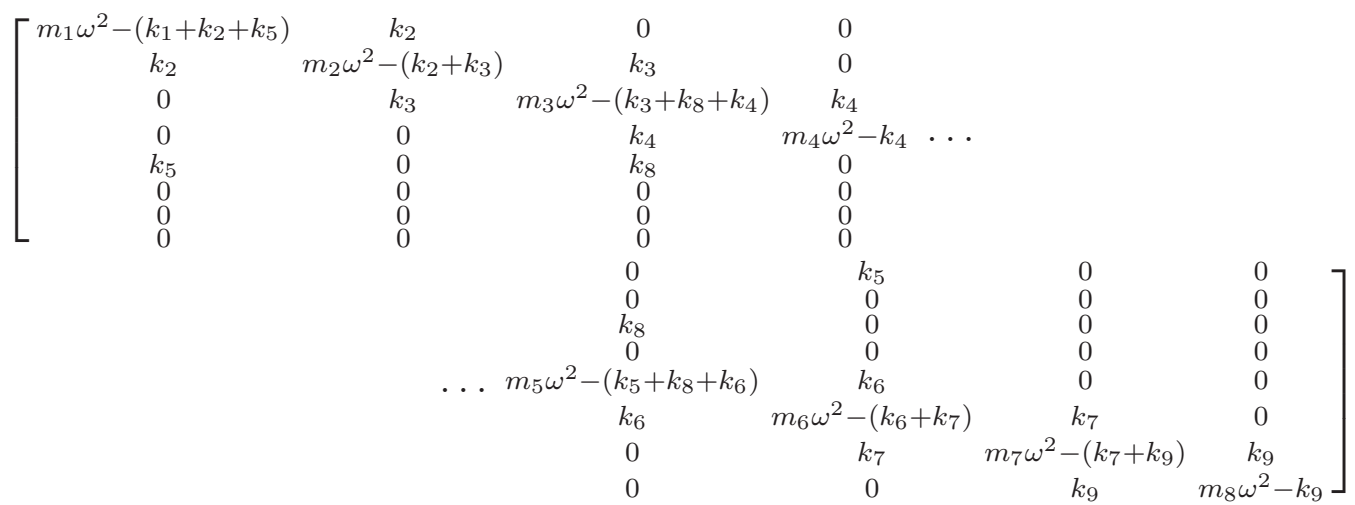




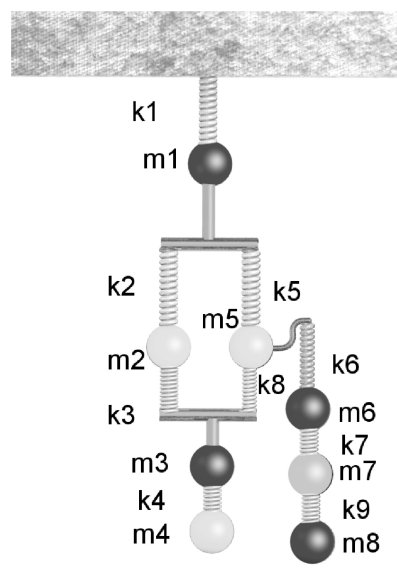

Figure 2: Sketch of the discrete system with eight masses.

\begin{tabular}{ccccccccc} 
matrix & 1 & 2 & 3 & 4 & 5 & 6 & 7 & 8 \\
\hline D & 355.9 & 425.4 & 491.2 & 503.3 & 675.2 & 720.6 & 827 & 1125 \\
A & 61.91 & 167.5 & 369.9 & 489.5 & 542.9 & 743.4 & 953.3 & 1250
\end{tabular}

Table 1: Eigenfrequencies of matrices $\mathbf{D}$ and $\mathbf{A}$ (in $\mathrm{Hz}$ ).

The accuracy of the iterative procedure described in Section 3.5 is measured by means of the error parameter

$$
e=\frac{\left\|\mathbf{A}_{\text {approx }}^{-1} \mathbf{A}-\mathbf{I}\right\|_{\mathrm{F}}}{n}=\frac{\left\|\mathbf{S}_{j, m}(\mathbf{I}-\mathbf{T})-\mathbf{I}\right\|_{\mathrm{F}}}{n}
$$

where $\|\bullet\|_{F}$ is the Frobenius norm.

The reduced dimension of this toy problem allows a detailed study of its spectral properties. Their evolution with frequency $f$ (with $f$ a real number) is shown in Fig. 3 for the undamped case. A can always be inverted (rank equals $n=8$ ) except for the eigenfrequencies shown in Table 1. Matrix $\mathbf{T}$ is only singular if one node is completely detached from the others.

Another relevant aspect is the number of eigenvalues of the matrix $\mathbf{T}$ with modulus larger than one. In practice, it means that the spectral radius of matrix $\mathbf{T}$ is also larger than one and the Neumann series does not converge. In this system, it happens for a frequency range between $61.91 \mathrm{~Hz}$ and $1250 \mathrm{~Hz}$. Fig. 3 also provides information on the number of conjugate pairs of eigenvalues, linked with the modification of parameters of Section 3.3.1.

The results in Fig. 4 illustrate how the solution of the mechanical problem can be obtained by the modified series proposed in Section 3. This means that the solution can be computed as a linear superposition of paths. The error measure $e$, defined in Eq. (45), is shown for several series depending on the number of modified parameters $(0,4$ or 8$)$. The total length $m+1$ of the series is 13 .

The first thing to be noted is a strong correlation between the convergence of the series (small values of $e$ ) and the fact that the number of modified parameters is equal 

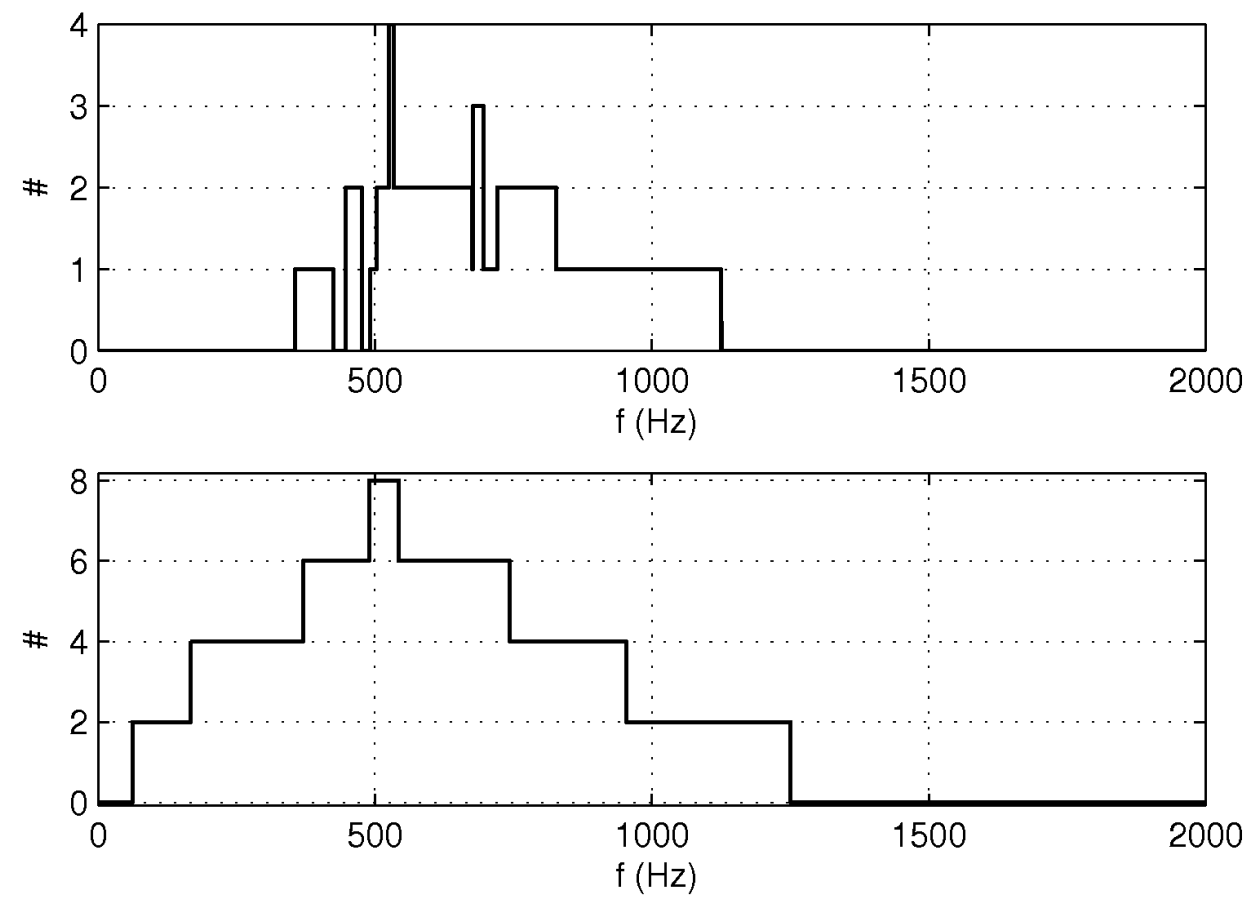

Figure 3: Description of the spectral properties of matrix $\mathbf{T}$ : number of conjugate pairs of eigenvalues (top); number of eigenvalues with modulus larger than one (bottom). Undamped case.

to or larger than the number of eigenvalues with modulus larger than one. See for example, the improvement obtained for the series with four modified parameters below $350 \mathrm{~Hz}$ and above $900 \mathrm{~Hz}$. This is in agreement with the fact that in this frequency range four eigenvalues of matrix $\mathbf{T}$ have modulus larger than one. When (all) eight parameters are modified, the convergence is improved in the whole frequency range. Note, however, that the error is large around the frequencies where $\mathbf{D}$ is singular. For larger frequencies all the series converge as expected, even if no parameter is modified. However, even modifying only some parameters largely reduces the error at high frequencies where $e$ has a very small value close to the numerical tolerance. The non-convergent frequencies are also improved.

The same mechanical system but with a hysteretic damping of $4 \%$ in all the springs is considered. Fig. 5 shows the spectral characteristics of the damped transfer matrix. Two important differences must be noted: i) the matrix is not singular at any real frequencies; ii) the eigenvalues are not complex conjugate. The frequency range where some eigenvalues have modulus larger than one is very similar to the undamped case.

Fig. 6 shows the influence of damping in the results. The general trend is similar to the undamped case. However, the effect of the eigenfrequencies of the matrices $\mathbf{D}$ and $\mathbf{A}$ is less important.

Fig. 7 shows a comparison between the exact value of the global transfer matrix 

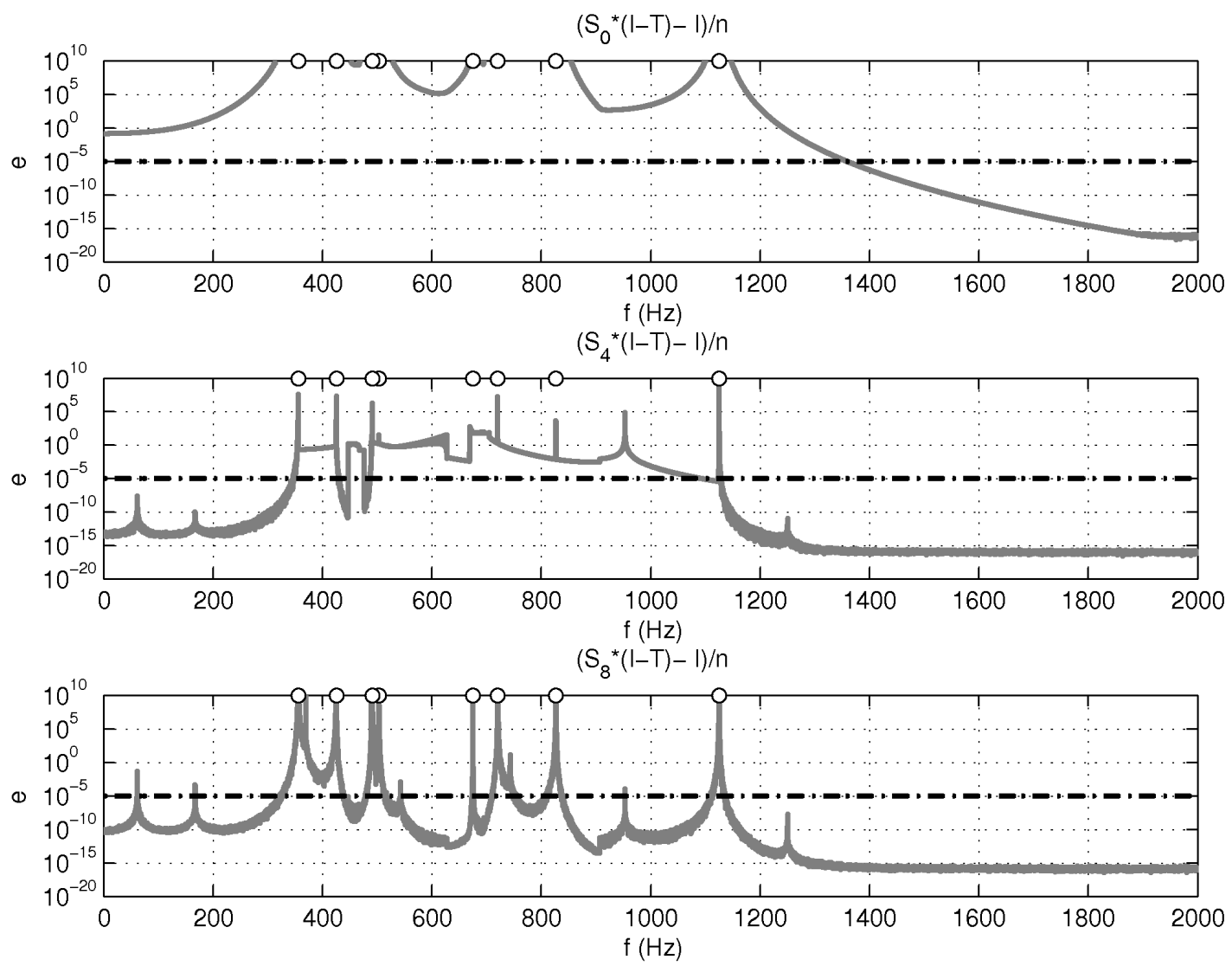

Figure 4: Difference between the exact solution and the solution obtained with the series. Influence of the number of modified parameters: 0 (unmodified Neumann series), 4 and 8 . The total length $m+1$ is 13 . The system is undamped.

$\mathbf{T}^{G}$ and the approximation obtained with the unmodified Neumann series and the Neumann series with all required correction parameters $(n=8)$. The coefficient $t_{14}^{G}$ is shown. It represents the displacement of mass number 4 for an unity force at mass 1. The exact value is obtained as detailed in [9]. The unmodified Neumann series diverges in the frequency interval where the spectral radius of the system matrix is larger than one. On the contrary, the modified Neumann series performs a very good reconstruction of the exact value. The inaccuracies of the modified series are mainly found at the frequencies where the matrix $\mathbf{D}$ is singular and the system is undamped. The error peaks around the eigenfrequencies of the mechanical system (matrix $\mathbf{A}$ singular) are smaller. Damping drastically reduces the error peaks of both types.

The error measure of Eq. (45) is global. However it is interesting to note that for most of the cases with large error, it is caused by only a few entries of the matrix $\mathbf{T}^{G}$ while the approximation of the others remains good enough. See for example Fig. 8 where only the coefficients with significant error have been highlighted with a grey square. 

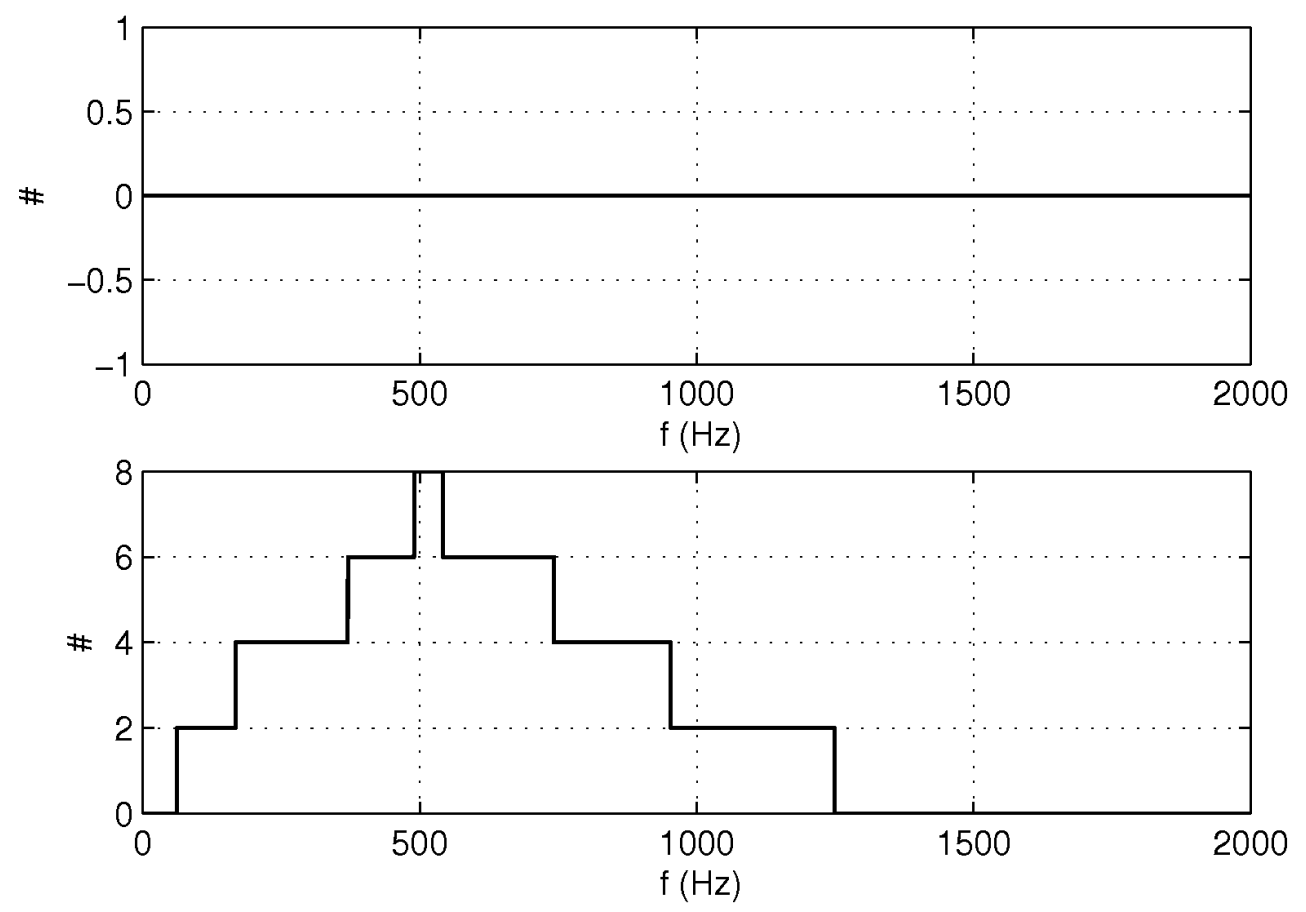

Figure 5: Description of the spectral properties of matrix $\mathbf{T}$ : number of conjugate pairs of eigenvalues (top); number of eigenvalues with modulus larger than one (bottom). With an hysteretic damping of $4 \%$ in all the springs.

\section{Conclusions}

The main interest of this research is theoretical and focused on the possibility of fully describing the solution of a mechanical problem by means of the superposition of transmission paths. It is conceived in the field of mechanics but the results, could be used in other fields of science because the framework of analysis is very general. It is only required that the problem remains linear, formulated in terms of a linear system of equations and where the path concept has some physical meaning.

In the current form, the final results cannot be taken as a calculation tool. Some aspects such as the matrix powers and series are very useful for theoretical proofs but may have some numerical drawbacks (i.e. rounding errors, large number of operations, filling of matrices). For this reason, a way to apply the main theoretical conclusions to the practical use in systems with a large number of unknowns is required. The algorithms can only be directly applied for calculation in mechanical systems with not many unknowns or in the low-frequency range. The applicability depends on the specific physical problem and the modelling technique used (it is not the same if the signals are the unknowns in every node of a FE mesh or just a few measurements in key points of a structure to generate a direct or global transfer matrix).

The main achievements of the work are the formal definition of path and the proof 

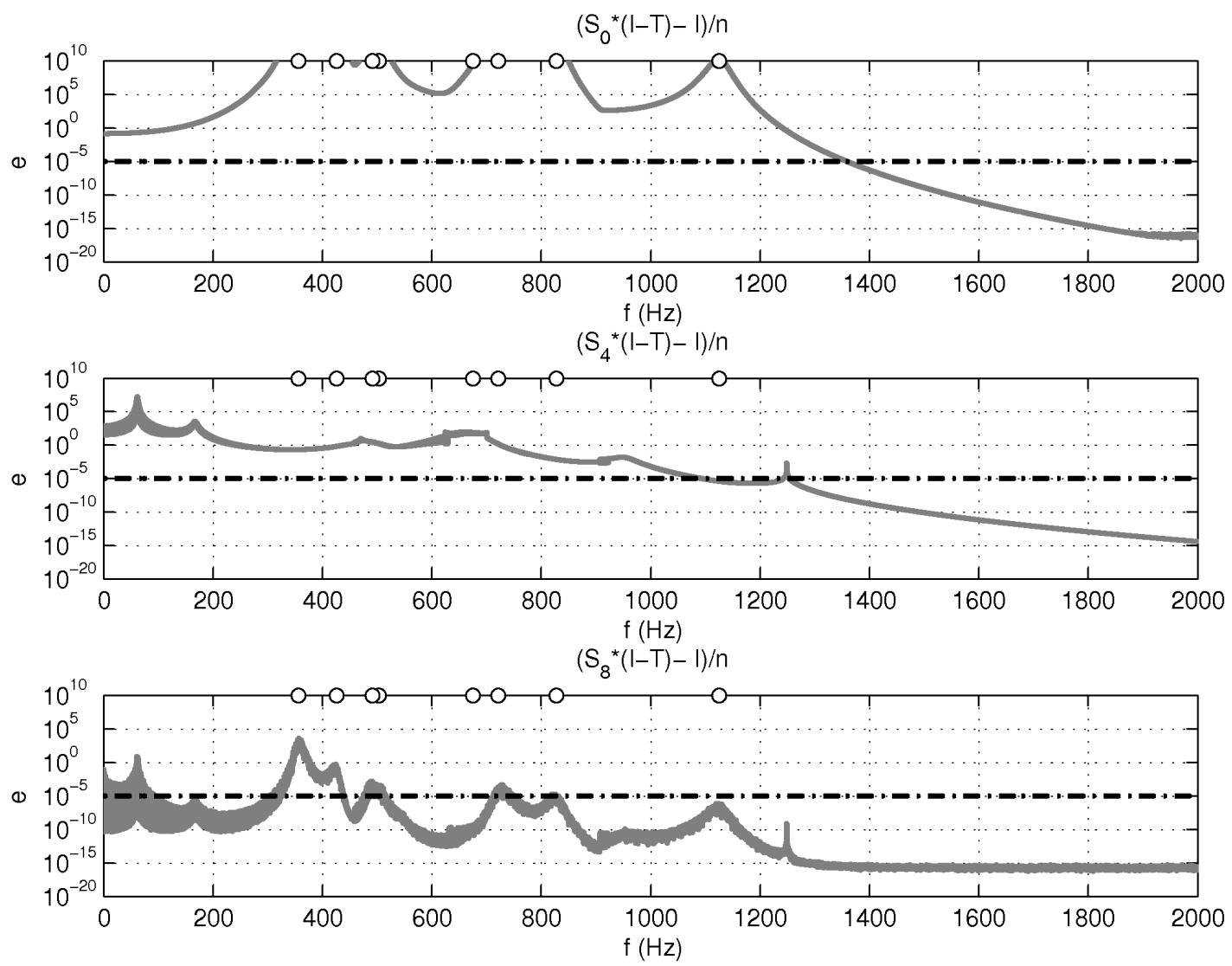

Figure 6: Difference between the exact solution and the solution obtained with the series. Influence of the number of modified parameters: 0 (unmodified Neumann series), 4 and 8 . The total length $m+1$ is 13 . The system has hysteretic damping of $4 \%$ in all the springs.

of completeness of the description of the system behaviour by means of paths. The former establishes a framework where other modelling techniques can be identified; it is not only valid when working with transfer matrices. The latter leads to a generalisation of the Neumann series that converges whatever the spectral radius of the transfer matrix is. Moreover, a simplified recursive expression of the generalised series is provided.

The theoretical findings presented here are important by themselves because it was not clear that any solution of a mechanical system could be described by means of paths. Moreover, they also open a door for improvement of simulation techniques in mechanics. 


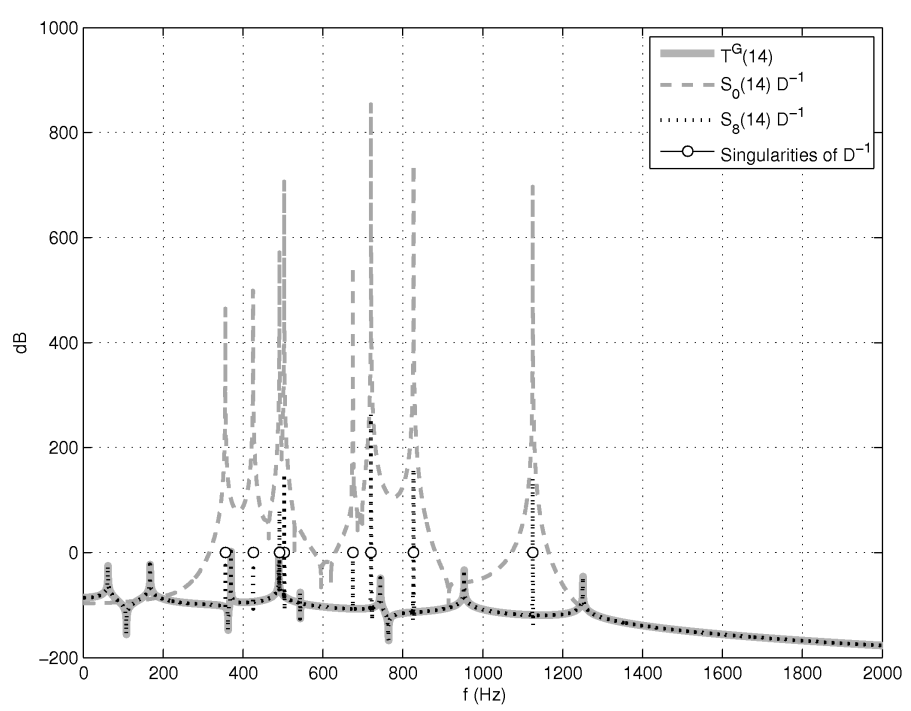

(a)

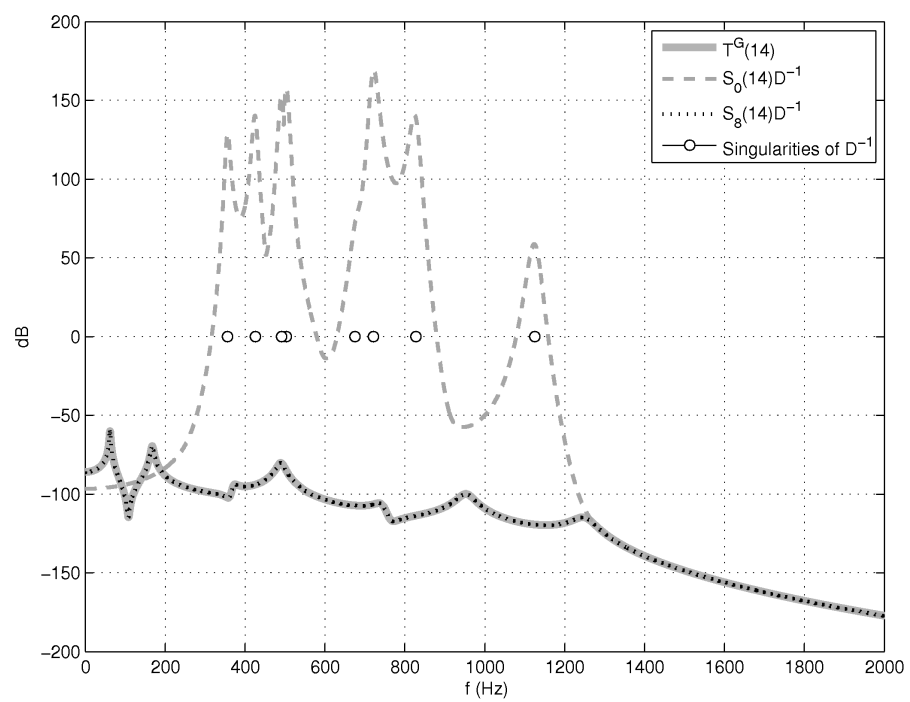

(b)

Figure 7: Value of the entry $t_{14}$ of the global transfer matrix $\mathbf{T}^{G}$. Comparison between: exact computation; approximation obtained with the unmodified Neumann series; and approximation obtained with the modified Neumann series including all the required correction parameters $(n=8)$ : (a) undamped mechanical system; (b) mechanical system with hysteretic damping of $4 \%$ in all the springs.

\section{A A link with iterative solvers: the acceleration of the Jacobi method}

The Jacobi method [33] for the iterative solution of linear systems of equations is:

$$
\mathbf{x}_{k+1}=\mathbf{D}^{-1} \mathbf{b}+\mathbf{D}^{-1}(-\mathbf{L}-\mathbf{U}) \mathbf{x}_{k}
$$




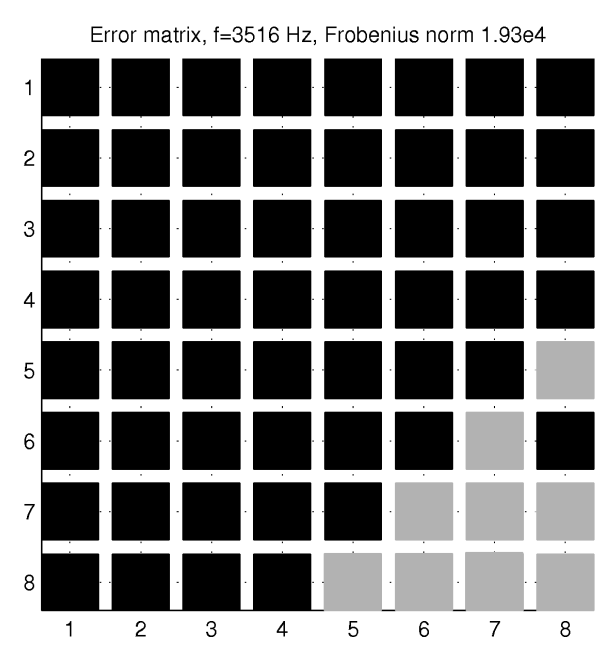

(a)

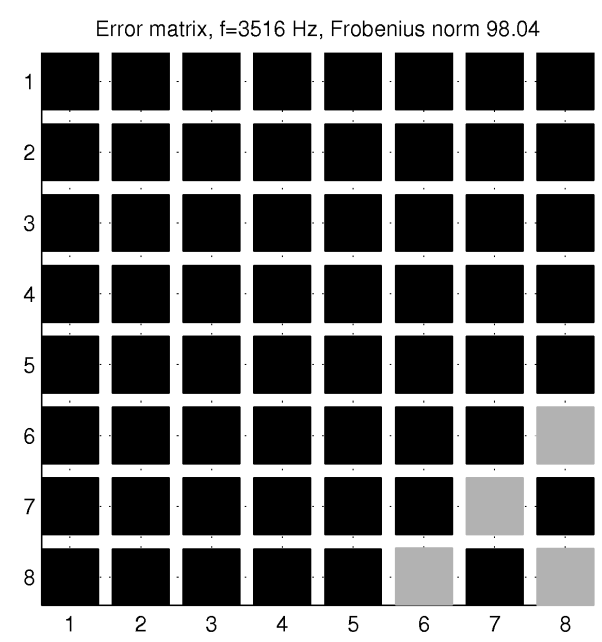

(b)

Figure 8: Representation of the 64 global transfer matrix $\mathbf{T}^{G}$ coefficients with an error larger than one (grey squares) in the comparison between the exact computation and approximation obtained with the modified Neumann series. Reconstruction of the matrix done for a frequency of $351.6 \mathrm{~Hz}$ : (a) Undamped mechanical system; (b) The mechanical system has hysteretic damping of $4 \%$ in all the springs.

It can be seen how its algebraic structure is the same as Eq. (5) and $\mathbf{T}$ plays the role of iteration matrix. A parallelism between the developed theory in the frame of the GTDT and the Jacobi method can be established.

The approximation $\mathbf{x}_{k+1}$ at the $k$ iteration in the Jacobi method can be obtained directly from a Neumann series like in Eq. (10). So, the correction of the series proposed in Section 3.2 can be applied in order to modify the Jacobi iteration algorithm.

The method is applied to the solution of linear systems with matrices of dimension 
300 that have the second largest eigenvalue not close to one. So, the discussion can be done by comparison of the standard Jacobi method with the proposed modification with only one parameter. As an example, see the results in Fig. 9. There is a comparison between the conventional Jacobi method and its modification, taking into account the dominant eigenvalue in a one-parameter correction. The solution obtained with a direct solver is used as reference in order to compute the relative error. We can see how the correction always accelerates the convergence. This is more relevant when the dominant eigenvalue is close to one because in that case, the convergence of the Jacobi method is very slow and it makes the practical applicability of the solver very poor.

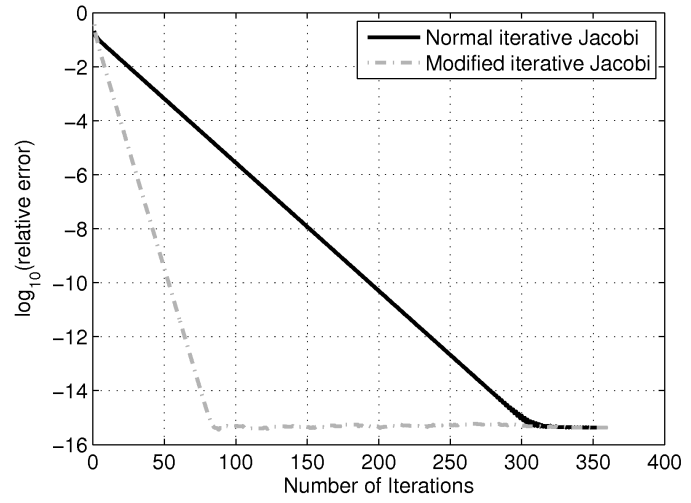

(a)

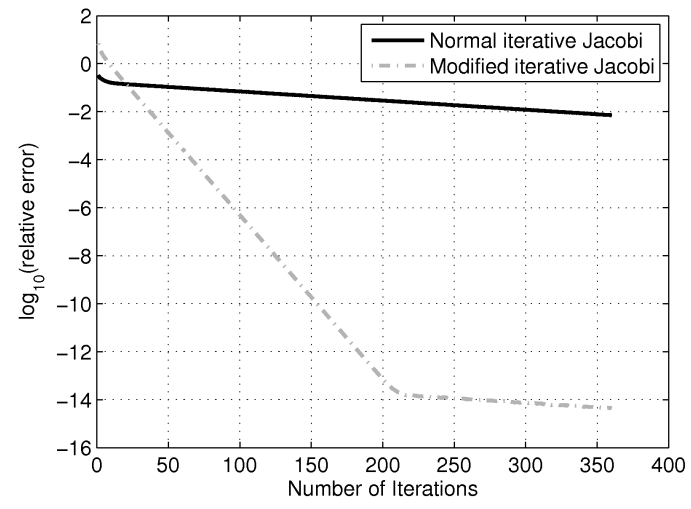

(b)

Figure 9: Convergence of the Jacobi method compared with its one-parameter modification. The dimension of the matrix is 300 and the two eigenvalues of largest modulus are:(a) $\left|\lambda_{1}\right|=0.89012$ and $\left|\lambda_{2}\right|=0.66415 \mid$; (b) $\left|\lambda_{1}\right|=0.99129$ and $\left|\lambda_{2}\right|=0.78518$.

\section{Acknowledgements}

LaCàN research group is grateful for the sponsorship/funding received from Generalitat de Catalunya (Grant number 2014-SGR-1471). 


\section{References}

[1] L.L. Koss and R.J. Alfredson. Multiple input correlation theory and the least squares method for the analysis of transient sound. J. Sound Vibr., 32(3):423427, 1974.

[2] J.S. Bendat. System identification from multiple input/output data. J. Sound Vibr., 49(3):293-308, 1976.

[3] J.S. Bendat. Solutions for the multiple input/output poblem. J. Sound Vibr., 44(3):311-325, 1976.

[4] R. Potter. Matrix formulation of multiple and partial coherence. J. Acoust. Soc. Am., 61(3):776-781, 1977.

[5] R.J. Alfredson. The partial coherence technique for source identification on a diesel engine. J. Sound Vibr., 55(4):487-494, 1977.

[6] L.L. Koss and R.J. Alfredson. Identification of transient sound sources on a punch press. J. Sound Vibr., 34(1):11-33, 1974.

[7] M. Lohrmann. Operational transfer path analysis: Comparison with conventional methods. J. Acoust. Soc. Am., 123(5):3534-3534, 2008.

[8] D De Klerk and A Ossipov. Operational transfer path analysis: Theory, guidelines and tire noise application. Mech. Syst. Signal Proc., 24(7):1950-1962, 2010.

[9] F. X. Magrans. Method of measuring transmission paths. J. Sound Vibr., 74(3):321-330, 1981.

[10] F. X. Magrans and O. Guasch. The role of the direct transfer matrix as a connectivity matrix and application to the Helmholtz equation in 2D: Relation to numerical methods and free field radiation example. Journal of Computational Acoustics, 13(02):341-363, 2005.

[11] O. Guasch and F. X. Magrans. The global transfer direct transfer method applied to a finite simply supported elastic beam. J. Sound Vibr., 276(12):335-359, 2004.

[12] J. Sapena, A. Tabbal, J. Jovié, and F. Guerville. Interior noise prediction in highspeed rolling stock drivers cab: Focus on structure-borne paths (mechanical and aero sources). In Tatsuo Maeda, Pierre-Etienne Gautier, CarlE. Hanson, Brian Hemsworth, JamesTuman Nelson, Burkhard Schulte-Werning, David Thompson, and Paul de Vos, editors, Noise and Vibration Mitigation for Rail Transportation Systems, volume 118 of Notes on Numerical Fluid Mechanics and Multidisciplinary Design, pages 445-452. Springer Japan, 2012.

[13] Nikos Zafeiropoulos, Andy Moorhouse, Andrew Mackay, and Uday Senapati. A comparison of two in-situ transfer path analysis methods. In Recent Advances in Structural Dynamics, 2013. 
[14] O. Guasch, C. García, J. Jové, and P. Artís. Experimental validation of the direct transmissibility approach to classical transfer path analysis on a mechanical setup. Mech. Syst. Signal Proc., 37(12):353-369, 2013.

[15] M. Van Der Seijs, D. de Klerk, and D. J. Rixen. General framework for transfer path analysis: History, theory and classification of techniques. Mech. Syst. Signal Proc., 2015.

[16] E. Luzzato and E. Ortola. The characterization of energy flow paths in the study of dynamic systems using S.E.A. theory. J. Sound Vibr., 123(1):189-197, 1988.

[17] R. Craik. Sound transmission paths through a statistical energy analysis model. Appl. Acoust., 30:45-55, 1990.

[18] F. X. Magrans. Definition and calculation of transmission paths within an S.E.A. framework. J. Sound Vibr., 165(2):277-283, 1993.

[19] A. Aragonès, L. Maxit, and O. Guasch. A graph theory approach to identify resonant and non-resonant transmission paths in statistical modal energy distribution analysis. J. Sound Vibr., 350:91-110, 2015.

[20] O. Guasch and A. Aragonès. Finding the dominant energy transmission paths in statistical energy analysis. J. Sound Vibr., 330(10):2325-2338, 2011.

[21] David Wilson. Prediction of bending wave transmission across coupled plates affected by spatial filtering and non-diffuse vibration fields. PhD thesis, University of Liverpool, 2014.

[22] J.W. Demmel. Applied Numerical Linear Algebra. EngineeringPro collection. Society for Industrial and Applied Mathematics (SIAM, 3600 Market Street, Floor 6, Philadelphia, PA 19104), 1997.

[23] L.N. Trefethen and D. Bau. Numerical Linear Algebra. Society for Industrial and Applied Mathematics, 1997.

[24] F. X. Magrans. Direct transference applied to the study of room acoustics. J. Sound Vibr., 96(1):13-21, 1984.

[25] O. Guasch and L. Cortés. Graph theory applied to noise and vibration control in statistical energy analysis models. J. Acoust. Soc. Am., 125(6):3657-3672, 2009.

[26] F. Bessac. Investigating the vibroacoustic behaviour of coupled systems through the coupling eigenvalues and eigenvectors. Theses, INSA de Lyon, May 1996.

[27] S. Finnveden. A quantitative criterion validating coupling power proportionality in statistical energy analysis. J. Sound Vibr., 330(1):87-109, 2011.

[28] P. Gajdatsy, K. Janssens, Wim Desmet, and H. Van der Auweraer. Application of the transmissibility concept in transfer path analysis. Mech. Syst. Signal Proc., 24(7):1963-1976, 2010. Special Issue: ISMA 2010. 
[29] C. Hopkins. Sound insulation. Routledge, 2012.

[30] J Jové and O Guasch. Mathematical link between direct transmissibility functions and the discrete characterisation of mechanical systems. In NOVEM2012: Sorrento (Italy, 1-4 April, Noise and Vibration: Emerging Methods, 2012.

[31] F. Ihlenburg. Finite element analysis of acoustic scattering. Springer, 1998.

[32] N Biggs. Algebraic graph theory. Cambridge University Press, 1993.

[33] Ph. G. Ciarlet, B. Miara, and J.-M. Thomas. Introduction to numerical linear algebra and optimisation. Cambridge University Press, 1989.

[34] Y Lebresne. Interventions practiques contre les bruits exterieurs des vehicles routiers. In Conferences Interkeller Gap (Zurich), number 11, pages 1-36, 1975. 